\section{The detection of rotation in random-dot patterns}

\author{
HERBERT H. BELL \\ Tennessee State University \\ Nashville, Tennessee 37203 \\ and \\ JOSEPH S. LAPPIN \\ Vanderbilt University \\ Nashville, Tennessee 37203
}

Previous research concerned with the detection of apparent motion in random-dot patterns has relied primarily upon rectangular patterns in which each dot within the pattern is shifted along the horizontal axis by a constant amount (e.g., Bell \& Lappin, 1973; Braddick, 1974; Lappin \& Bell, 1976). However, such translations represent only one of the possible rigid transformations associated with the motion of an object in the environment. Despite the commonness of these other rigid transformations, very little is known about their detectability in randomdot patterns.

The purpose of the present study was to determine the ability of observers to detect rotation within two-dimensional random-dot patterns. The randomdot patterns used in the present experiment were defined in polar coordinates $(r, \theta)$ rather than Cartesian coordinates $(x, y)$. Rotation of these circular patterns about their origin involves a different transformation from that employed in our earlier research (Bell \& Lappin, 1973; Lappin \& Bell, 1976). While a translation displaces each dot the same distance in the same direction, a rotation displaces the dots varying distances in varying directions (as defined in Cartesian coordinates), depending on their positions in the pattern. Because each dot undergoes a slightly different displacement, the detectability of global rotations of these patterns is uncertain. Evidence that rotational transformations of these patterns might be difficult to detect was provided by Julesz and Hesse (1970), who showed that subsets of a textured pattern composed of thousands of elements could not be spontaneously discriminated solely on the basis of differences in the direction of rotations. The patterns in the present experiments

This research was supported by Public Health Service Grant MH 21105 and NSF Grant BNS 78-05857 to Joseph S. Lappin. The authors gratefully acknowledge the programming assistance provided by Melanae Schnurr. Requests for reprints may be sent to Herbert H. Bell, Department of Psychology, Tennessee State University, Tenth and Charlotte Avenues, Nashville, Tennessee 37203. differ, however, in that the same transformation with the same center of rotation is applied to all of the component elements, whereas in the Julesz and Hesse experiment each component was rotated about its geometric center.

In our previous experiments employing translations, we found the detectability of motion to be inversely related to both the interstimulus interval (ISI) and the relative extent of displacement between the two successive displays (Bell \& Lappin, 1973; Lappin \& Bell, 1976). We manipulated these same variables in the present investigation in order to determine whether or not rotational motion could be detected and whether these variables also affected the detection of rotational motion. As described below, the results of this investigation are very similar to our earlier experiments involving translations.

\section{Method}

Each stimulus presentation consisted of a pair of circular randomdot patterns, separated by an ISI of $0,50,150$, or $300 \mathrm{msec}$ (see Figure 1). The two successive patterns forming the stimulus were identical, except that the second pattern had been rotated about its origin by $1,2,3$, or 4 radii ( 6 to $24 \mathrm{deg}$, respectively). The relation between the individual dots in each pair remained constant-the only difference between the two successive patterns was their rotation about the origin.

A digital computer (PDP-8/1) controlled the presentation of each pattern on a CRT display scope (Tektronix 604) equipped with a rapidly decaying phosphor (P-15). Each pattern contained 480 dots randomly positioned along 60 equally spaced imaginary radii defining the circular patterns. Each radius contained eight dots, with each dot randomly assigned to one of 16 possible locations. The dots were sequentially plotted with an interval of $20 \mu \mathrm{sec}$ between successive dots, the entire display being successively replotted until an exposure duration of $150 \mathrm{msec}$ was reached. The stimuli were binocularly viewed ${ }^{1}$ in a dimly lit room from a distance of approximately $127 \mathrm{~cm}$; they subtended visual angles of $2.3,4.6$, or $9.2 \mathrm{deg}$. These visual angles were obtained by varying the spacing between the dots, and thus the diameter

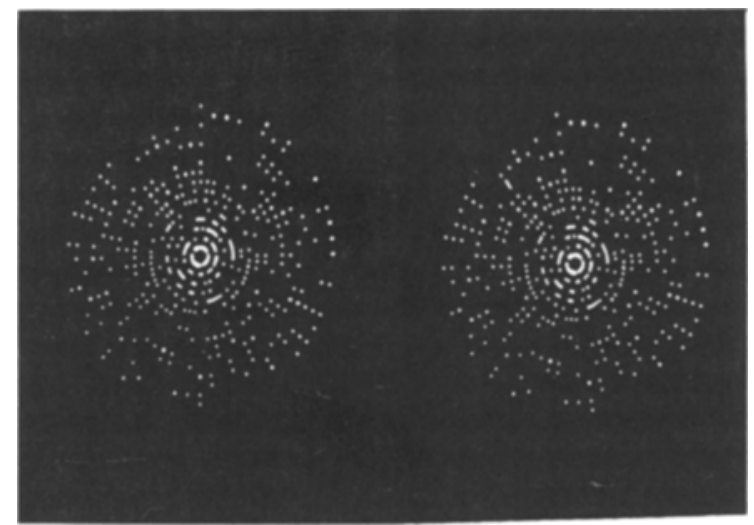

Figure 1. A stimulus pattern consisting of a pair of sequentially presented random-dot patterns. The stimuli are identical except for the rotation of the entire pattern about the origin. 
of the circular patterns, while keeping the size of the individual dots constant.

Three paid observers who had served in similar experiments participated in six experimental sessions. ${ }^{2}$ Within each session, the visual angle was held constant while the ISI, direction of rotation, and relative extent of rotation (in number of radii) were randomly varied. The ordering of the visual angles was counterbalanced across observers. Each session consisted of 400 trials and required about $\mathbf{3 0} \mathrm{min}$ to complete. The observer initiated each trial by depressing two response keys. Releasing the appropriate response key identified the direction of rotation as clockwise or counterclockwise. An auditory signal followed each correct discrimination.

\section{Results and Discussion}

The results of this experiment are summarized in the left-hand panel of Figure 2, which gives the percentage of correct discriminations as a function of the relative extent of rotation and the ISI. These percentages have been averaged across the visual angles $[F(2,4)=1.6, p<.3]$ and directions of rotation $[F<1.0]$ because these variables had no reliable effect on discrimination accuracy and no interaction with the other variables.

An inspection of the left-hand panel in Figure 2 clearly shows that both the ISI and the relative extent of the rotation were inversely related to discrimination accuracy. The effects of these variables were statistically tested using a repeated-measures analysis of variance with ISI, relative extent of rotation, direction of rotation, and visual angle as fixed factors and observers as a random factor. Statistically significant main effects were found for relative extent of rotation $[F(3,6)=34.6, p<.001]$ and ISI $[F(3,6)=20.9, p<.01]$. The only significant interaction was between relative extent of rotation and ISI $[F(9,18)=19.4, p<.001]$. All other main effects and interactions were nonsignificant at the .05 level.

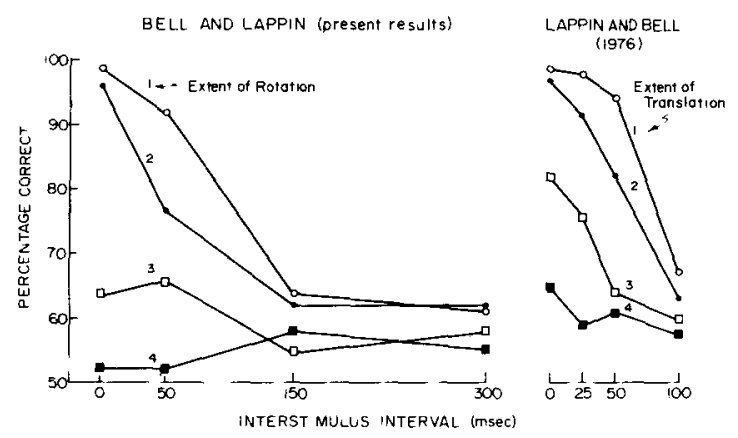

Figure 2. The left-hand panel shows the percentage of correct discriminations for each relative extent of rotations as a function of interstimulus interval. The right-hand panel presents comparison data for translations from "The Detection of Coherence in Moving Random-Dot Patterns" by J. S. Lappin and H. H. Bell (Vision Research, 1976, 16, 161-168. (Copyright 1976 by Pergamon Press. Reprinted by permission.)
For the purpose of comparison with our earlier experiments involving translations, a portion of the data from Lappin and Bell (1976) is presented in the right-hand panel of Figure 2. A visual comparison of these two panels clearly indicates that the effects of ISI and relative extent of rotation found in this experiment closely parallel those found in our earlier experiments involving translation of rectangular patterns: Percentage of correct discriminations decreases as the angle of rotation increases, just as with increases in the extent of translation; percentage of correct discriminations decreases as ISI increases, both for rotation and translation. In addition, the absence of any significant effect for the visual angle subtended by the pattern supports our earlier finding that the relative extent of displacement of a pattern is more important than the absolute displacement across the retina (Bell \& Lappin, 1973; Lappin \& Bell, 1976). Although this experiment did not directly compare the detectability of rotations and translations, ${ }^{3}$ the fact that performance is similar and is influenced by the same variables in essentially the same manner suggests that the perceptual processes underlying the detection of coherent motion are the same regardless of whether that motion is based upon the rotation or translation of the individual pattern elements.

Several aspects of these findings are difficult to reconcile with the hypothesis that motion detection derives from the operation of special-purpose mechanisms selectively turned to specific directions and velocities (e.g., Barlow, Hill, \& Levick, 1964): First, it is unclear how the globally coherent relationships in these random-dot patterns would be detected by specific motion analyzers focusing upon the motion of individual elements-particularly in the case of rotations, where the distances and directions of motion are heterogeneous. Second, in contrast to claims by Braddick (Braddick, 1974; Braddick \& Adlard, 1978), the detectability of the global motion of these random-dot patterns has been found in both this and our previous experiments to be limited by relative distance of displacement measured in relation to the spacing of components in the pattern rather than by absolute distance of displacement measured in relation to the retina.

These results seem incompatible with a neural process composed of specifically tuned motion analyzers, but compatible with one that detects the coherent organization of patterns in time and space. One such process is the cross-correlation of two successive random-dot patterns (Lappin \& Bell, 1976; Uttal, 1975). The perception of global motion would depend on the detection of the coherent relationship between the two successive patterns. 


\section{REFERENCES}

Barlow, H. B., Hill, R. M., \& Levick, W. R. Retinal ganglion cells responding selectively to direction and speed of image motion in the rabbit. Journal of Physiology, 1964, 173, $377-404$.

Bell, H. H., \& Lappin, J. S. Sufficient conditions for the discrimination of motion. Perception \& Psychophysics, 1973, 14, 45-50.

BraDdick, O. A short-range process in apparent motion. Vision Research, 1974, 14, 519-527.

BradDick, O., \& Adla RD, A. Apparent motion and the motion detector. In J. C. Armington, J. Krauskopf, \& B. R. Wooten (Eds.), Visual psychophysics and physiology. New York: Academic Press, 1978.

Julesz, B., \& Hesse, R. I. Inability to perceive the direction of rotation movement of line segments. Nature, 1970, 225, 243-244.

LAPpin, J. S., \& BELl, H. H. The detection of coherence in moving random-dot patterns. Vision Research, 1976, 16, 161-168.
UTTAL, W. R. An autocorrelation theory of form detection. Hillsdale, N.J: Erlbaum, 1975.

\section{NOTES}

1. Additional control experiments found no differences between monocular and binocular viewing for this particular task.

2. A separate control experiment comparing the performance of experienced and naive observers found no reliable differences in performance.

3. While it may be of interest to directly compare the detectability of rotations and transformations, any observed difference may be due to the unique combination of parameters selected. We believe that the more important point is that the detectability of both rotations and transformations appears to depend upon the same variables in approximately the same manner.

(Received for publication April 24, 1979; revision accepted September 13, 1979.) 